A dementing illness changes the carers as well. They have to come to terms with the effects of the illness and learn to accept deteriorating standards and to take risks. Setting the house on fire, wandering in the street and being run over, and dying when lost in the park are all recognised risks and therefore genuine worries. The major task of the new breed of psychiatrists in caring for patients with dementia will thus be in family and neighbourhood therapy. What they must, however, ensure is that in defending the line of diminishing institutional resources they do not push people too far. The ultimate protest of someone who cannot cope any longer is murder. Many of our present services give priority to the elderly who live alone; in reality those who live with others may cause the greatest stress. In developing a policy of home care for the demented we must care for the carers. The staff in residential care and in long-stay hospitals will also require training and support.

The college report is timely; it should be essential reading for all, for all must realise that a policy of coping with demented people at home implies that at times of illness the families will not be able to cope and will inevitably turn to us for help. Let us hope that such help will not be denied.

P H MILlaRD

Eleanor Peel Professor of Geriatric Medicine,

St George's Hospital Medical School,

London SW17 0QT

1 College Committee on Geriatrics of the Royal College of Physicians. Organic mental impairment in the elderly. $f R$ Coll Physicians Lond 1981;15:141-67.

2 Pickering T. The future of cardiology and psychogeriatrics. $\mathrm{Br} \mathrm{Med} \mathcal{F}$ $1981 ; 283: 377$.

${ }^{3}$ Pitt B. The future of cardiology and psychogeriatrics. $\mathrm{Br} \mathrm{Med} \mathcal{F} 1981$; $283: 494-5$.

4 Godber $\mathrm{C}$. The future of cardiology and psychogeriatrics. $\mathrm{Br} \mathrm{Med} \mathcal{F}$ $1981 ; 283$ :495.

5 Post F. The future of cardiology and psychogeriatrics. Br Med $\mathcal{F} 1981$; 283:496.

6 Wattis J, Wattis L, Arie T. Psychogeriatrics: a national survey of a new branch of psychiatry. Br Med F 1981;282:1529-33.

${ }^{7}$ Smith JS, Kiloh LG. The investigation of dementia: results in 200 consecutive admissions. Lancet 1981 ; :824-7.

${ }^{8}$ Oram JJ, Edwardson J, Millard PH. Investigation of cerebrospinal fluid neuropeptides in idiopathic senile dementia. Gerontology 1981 ;27: 216-23.

9 Eastwood R, Corbin S. Investigation of suspect dementia. Lancet 1981 ; : 1261 .

\section{Cutaneous mycobacteriosis}

Skin lesions caused by mycobacteria are comparatively rare, and few clinicians other than dermatologists have sufficient experience to make a confident diagnosis. Their very rarity, however, demands a high index of suspicion, for lesions may otherwise remain undiagnosed for many years. The nomenclature of these lesions-tuberculosis colliquativa cutis and tuberculosis cutis miliaris acuta generalisata are good examples -must deter all but the most ardent classicists. Furthermore, the range of mycobacterial diseases is changing and several revisions have recently been made of Runyon's classification ${ }^{1}$ based on colonial morphology, reaction to light, and rates of growth. ${ }^{2}$

Fortunately for baffled clinicians, Beyt and his colleagues have recently proposed a relatively simple classification of the diverse forms of cutaneous mycobacterial infections. ${ }^{3}$ The first group includes all the lesions caused by inoculation from an exogenous source. In patients not previously exposed to mycobacteria the primary cutaneous focus is associated with regional lymphadenitis to form the primary complex. This pattern is seen most commonly in children: the tuberculin test result becomes positive, the nodes may ulcerate, and rarely the disease may progress to acute miliary tuberculosis. In patients who have been infected before, on the other hand, the cutaneous lesion results in a hyperkeratotic papule without adenopathy-the "prosector's wart." Six out of 10 of such lesions reported in the past 10 years have been in people working in medically related professions.

The second group includes all lesions from an endogenous source. The most common manifestation is scrofulodermathe fistulous openings of sinuses originating in glands or bones previously infected with mycobacteria, as described by Michelson in 1924. ${ }^{4}$ This condition is now rare but may still be seen when lesions in lymph nodes, the epididymis, or bone form local abscesses which drain through the skin with the formation of sinuses. The other endogenous source is tuberculous material passed through body orifices-orificial cutaneous tuberculosis. Oral, perirectal, and labial lesions may remain undiagnosed for many years. ${ }^{5}$ Tuberculous ischiorectal abscesses may sometimes be haematogenous.

Lesions due to haematogenous spread, indeed, form the third group: the main conditions are lupus vulgaris, acute haematogenous cutaneous lesions, and multiple nodules or abscesses. In lupus vulgaris, plaques and nodules (usually on the face) ulcerate and scar and contain "apple-jelly" nodules. Extensive scarring causes serious disfigurement. Tubercle bacilli are sparse in the lesions and may have attenuated virulence. In his study of 3902 patients, Horowitz found evidence of tuberculous disease elsewhere in $76 \%$ - usually in lymph nodes, less commonly in the respiratory system and skeleton. ${ }^{6}$ Chemotherapy is highly effective, though the problem of scarring remains. The rare but potentially fatal acute haematogenous lesions are characterised by multiple cutaneous pustules in which vascular thrombi contain numerous bacilli.?

Many cutaneous lesions, particularly those caused by inoculation from an exogenous source, are due to mycobacteria other than tubercle bacilli. Mycobacterium marinum (balnei) is frequently responsible for ulcerated lesions appearing two to three weeks after exposure to trauma sustained on beaches, in swimming pools, or in aquariums. Skin lesions may be caused by many other mycobacteria and may occur in unexpected clinical settings, such as wound infection after open-heart surgery ${ }^{8}$ and in the recipients of renal homografts. ${ }^{9}$ Necrotic indolent ulcers on the legs of children in Africa and Australia may be caused by $M$ ulcerans. ${ }^{10} 11$

Beyt and his colleagues ${ }^{3}$ specifically excluded from their classification the tuberculids such as erythema induratum and papulonecrotic tuberculid. These are considered to be hypersensitivity phenomena; their histological features include caseation necrosis with the formation of giant cells and associated perivascular infiltrate. The result of the tuberculin test is always strongly positive, but stains and cultures of the lesions do not yield mycobacteria. Another omission from the classification is the cutaneous complications of BCG vaccination. ${ }^{12}$

When a patient has a skin lesion that could be mycobacterial investigation should not be confined to histological examination and a tuberculin test, which may give a negative result to purified protein derivative in some infections. Culture is important, so that the causative mycobacteria may be identified. Many of the mycobacteria other than tubercle bacilli are 
resistant to most of the commonly used antituberculosis drugs. In $M$ kansasii infections, however, treatment with three drugs is often effective, the in-vitro drug resistance being of no prognostic importance. Amikacin, doxycycline, or cotrimoxazole may possibly be of benefit in $M$ marinum infections.

N W HORNE

Consultant Physician,

Chest Unit,

City Hospital,

Edinburgh EH10 5SB

1 Timpe A, Runyon EH. The relationship of "atypical" acid-fast bacteria to human disease: a preliminary report. $\mathcal{F}$ Lab Clin Med 1954 ;44:202-9.

2 Wolinsky E. Nontuberculous mycobacteria and associated diseases. Am Rev Respir Dis 1979;119:107-59.

${ }^{3}$ Beyt BE, Ortbals DW, Cruz DJS, Kobayashi GS, Eisen AZ, Medoff G. Cutaneous mycobacteriosis: analysis of 34 cases with a new classification of the disease. Medicine $1981 ; 60: 95-109$.

${ }^{4}$ Michelson HE. Scrofuloderma gummosa (tuberculosis colliquativa). Archives of Dermatology and Syphilology 1924;10:565-78.

5 Millar JW, Holt S, Gilmour HM, Robertson DHH. Vulval tuberculosis. Tubercle $1979 ; 60: 173-6$.

${ }^{6}$ Horowitz O. Lupus vulgaris cutis in Denmark, 1895-1954. Its relation to the epidemiology of other forms of tuberculosis. Epidemiology and course of the tuberculosis infection based on 3902 cases from the Finsen Institute, Copenhagen. Acta Tuberculosea Scandinavica 1960; suppl 49:1-37.

7 Schermer DR, Simpson CG, Haserick JR, van Ordstrand HS. Tuberculosis cutis miliaris acuta generalisata. Report of a case in an adult and review of the literature. Arch Dermatol 1969;99:64-9.

${ }^{8}$ Robicsek F, Daugherty HK, Cook JW, et al. Mycobacterium fortuitum epidemics after open-heart surgery. $\mathcal{F}$ Thorac Cardiovasc Surg 1978; $75: 91-6$.

${ }^{9}$ Graybill JR, Silva J Jr, Fraser DW, Lordan R, Rogers E. Disseminated mycobacteriosis due to Mycobacterium abscessus in two recipients of renal homografts. Am Rev Respir Dis 1974;109:4-10.

10 Uganda Buruli Group. Clinical features and treatment of pre-ulcerative

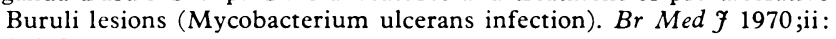
390-3.

${ }^{11}$ Radford AJ. Mycobacterium ulcerans in Australia. Aust NZ $\mathcal{f}$ Med $1975 ; 5: 162-9$.

12 Dostrovsky A, Sagher F. Dermatological complications of BCG vaccination. Br F Dermatol 1963;75:181-92.

\section{Prolactinoma: a question of rational treatment}

The introduction of assays for human prolactin ${ }^{1}$ and the use of drugs such as bromocriptine for treating hyperprolactinaemia ${ }^{2}$ have had a major impact on the diagnosis and initial management of prolactin-secreting tumours. Many problems, however, remain. How common are prolactinomas? What is known of their pathophysiology? What tests can be used to distinguish reliably between prolactinomas and other causes of hyperprolactinaemia? What is the clinical course of the condition? Which patients require treatment? What type of treatment should be given? Only when these questions can be answered satisfactorily shall we be able to formulate a rational policy for long-term management.

Prolactin is principally under inhibitory control by dopamine secreted by the hypothalamus, ${ }^{3}$ so that in theory either a deficiency of dopamine or decreased sensitivity to it on the part of the pituitary lactotrophs could lead to the development of a prolactinoma. Studjes on the function of normal anterior pituitary tissue surrounding tumours suggest that there is increased hypothalamic secretion of dopamine, ${ }^{45}$ implying a reduced response of the tumour lactotrophs to dopamine as compared with that of the normal prolactin-secreting cells. ${ }^{6}$
The hyperprolactinaemia that results then stimulates the release of dopamine. ${ }^{7}$ Prolactinomas seem, then to develop as a primary pituitary disorder and not secondary to hypothalamic disease, so that selective surgical removal of the tumour, preserving anterior pituitary function (microadenomectomy), ${ }^{8}$ may restore normal hypothalamic control. ${ }^{910}$

Early studies ${ }^{11}$ suggested that most women with prolactinomas would present in early reproductive life with amenorrhoea and infertility. In fact, any type of menstrual abnormality may be associated with hyperprolactinaemia, including dysfunctional uterine bleeding, regular anovulatory cycles, or cycles deficient in luteal-phase progesterone. ${ }^{12}$ The incidence of galactorrhoea is variable $(30-80 \%),{ }^{11}{ }^{13}$ depending to a large extent on the care taken by the clinician to examine the breasts and the meaning placed on traces of fluid expressed. Impairment of the visual fields due to an expanding tumour is fortunately rare (occurring in $7 \%$ of cases). ${ }^{11}$ In contrast, men present later (mean 39 years) with impotence $(90 \%)$ and obesity $(48 \%){ }^{14}{ }^{15}$ Galactorrhoea is unusual, but many patients have evidence of suprasellar expansion, often reflecting the delay in diagnosis of a prolactinoma as the cause of impotence. In both sexes the clinical and biochemical features of hypogonadism may be reversed by treatment which lowers serum concentrations of prolactin-provided that secretion of pituitary gonadotrophins is adequate. ${ }^{14} 15$

Hyperprolactinaemia is not always attributable to a prolactinoma: other possibilities include pregnancy, prolactinstimulating drugs, renal failure, and hypothyroidism. ${ }^{1316}$ When the serum concentration of prolactin is consistently high (mean of three samples above $2000 \mathrm{mU} / \mathrm{l}$ ) a confident diagnosis of a prolactin-secreting macroadenoma can be made if the pituitary fossa is obviously enlarged on plain skull radiographs. (An empty fossa syndrome must be excluded later.) Testing the visual fields and computed tomography are useful in defining the extent of the tumour, but the suprasellar region may be assessed more accurately by positive contrast (metrizamide) cisternography. ${ }^{17}$ Carotid angiography may be needed before surgery to ensure that the pituitary fossa is not occupied by an aneurysm. ${ }^{18}$ The effect of the tumour on the function of the rest of the pituitary should be assessed.

Macroadenomas that extend above the sella to affect the optic pathways have usually been decompressed by the transfrontal route with postoperative external irradiation to prevent recurrence ${ }^{19}$ and treatment with bromocriptine should hyperprolactinaemia persist. More recently treatment with bromocriptine has been shown to shrink pituitary tumours, ${ }^{20-23}$ and such treatment may therefore be an alternative to neurosurgery-even in patients with visual impairment-provided that special care is taken in monitoring response to treatment. Macroprolactinomas without any substantial suprasellar extension do not require transfrontal surgery but are usually too large to be "cured" by transsphenoidal microsurgery. 1024 Bromocriptine is the treatment of choice to lower prolactin concentrations and to restore fertility. While there is no evidence that bromocriptine is teratogenic, ${ }^{25} 26$ patients are usually advised to discontinue treatment as soon as pregnancy is confirmed. Such patients have a real risk (35\%) of expansion of the tumour during pregnancy. ${ }^{27}$ The risk can be kept to a minimum if the tumour is treated with external pituitary irradiation $^{26}$ or yttrium-90 implantation. ${ }^{28}$ Alternatively, an expectant policy may be justifiable, ${ }^{29}$ with monthly assessment of the visual fields and the serum concentrations of prolactin. Concentrations raised above the expected range for the stage of pregnancy ${ }^{16}$ or symptoms suggesting enlargement of the tumour are immediate indications for treatment with bromo- 